\title{
Taking the Code for a Walk
}

\author{
Delfina Fantini van Ditmar, Royal College of Art
}

Dan Lockton, Royal College of Art

Our lives are increasingly concept such as smartness then becomes informed by an relational, a property subjectively algorithmic paradigm. assessed by users through a particular We are profiled and interaction rather than something that analyzed, our behavior can be specified in itself.translated into data To explore an alternative to a purely and connected to algorithmic logic, we developed three larger bodies of data. But as technology practice-based experimental projects begins to make autonomous decisions, it is important to question the place of humans in algorithmic logic.

The logic reflected in the current technological landscape has implications for the interactions we have with our environment and our ways of living. In the dominant paradigm, however, human subjectivity is largely missing, or founded in simplistic assumptions without consideration of users' perspectives on data, contextual significance, and situated values.

How can interacting with these systems be more commensurate [1]? Acknowledging and accounting for the role of users in actively making sense of their own data is key. As discussed in second-order cybernetics, all knowledge is dependent on the observer's involvement [2]. Defined by Heinz von Foerster as "the study of observing systems," second-order cybernetics focuses on the observer as subject, aware of his or her own observing. As Ranulph Glanville put it, "When what is observed is observed by an observer, that observer is responsible for the observation, the sense he makes of it, and the actions he takes based on that sense" [3].

Second-order cybernetics, in highlighting how meaning is constructed, encourages us to acknowledge the importance of humans in making sense of data, not just as producers of data. A concept such as smartness then becomes relational, a property subjectively assessed by users through a particular interaction rather than something that can be specified in itself. To explore an alternative to a purely algorithmic logic, we developed three practice-based experimental projects around the idea of the smart home. By taking a second-order cybernetic approach, the projects explore a different perspective on the human experience of the Internet of Things (IoT) in practice, in relation to its background operation. 


\section{PROJECT 1: \\ WHAT THE KITCHEN THINKS IT KNOWS ABOUT YOU}

As part of Universities Week 2014 - a series of events about public engagement with academic research-we produced an interactive exhibition at the Natural History Museum, London, in conjunction with RCA doctoral candidate Mike Kann. Our aim was to explore public conceptions of domestic IoT, introducing the notion of a smart home and discussing people's ideas around it. After introducing IoT hardware such as sensors and microcontrollers (presented in an old museum cabinet), we provided visitors with a tangible IoT experience in which we asked them to make a hot drink, choosing from a range of options (e.g., decaffeinated coffee, black tea, soy milk, brown sugar, sweetener). After preparing their drinks, participants received a receipt with their choices, printed alongside suggestions for related products. These suggestions were taken from Amazon's recommendations. Many made sense (if you used soy milk, you probably like rice crackers) but others were less obviously connected (brown sugar was linked to tomato sauce).

Now that participants had experienced one way in which IoT technology could work in a smart home-having their behavior sensed in real time, perceiving their data being associated with a larger body of data, and experiencing assumptions made about them-we discussed people's sentiments toward this kind of datasharing and recommendation. Through a questionnaire based on scenarios of IoT applications in the kitchen, bathroom, and bedroom (Figure 1), we found that people's concerns, the benefits that people can see, and their willingness to share information change in different domestic spaces. This investigation provided a qualitative dataset of respondents' sentiments toward the implications of domestic IoT. One finding was that kitchens were where people felt most comfortable sharing their data. It was thus the kitchen on which the next project focused-specifically the fridge, as the archetypal IoT-connected device.
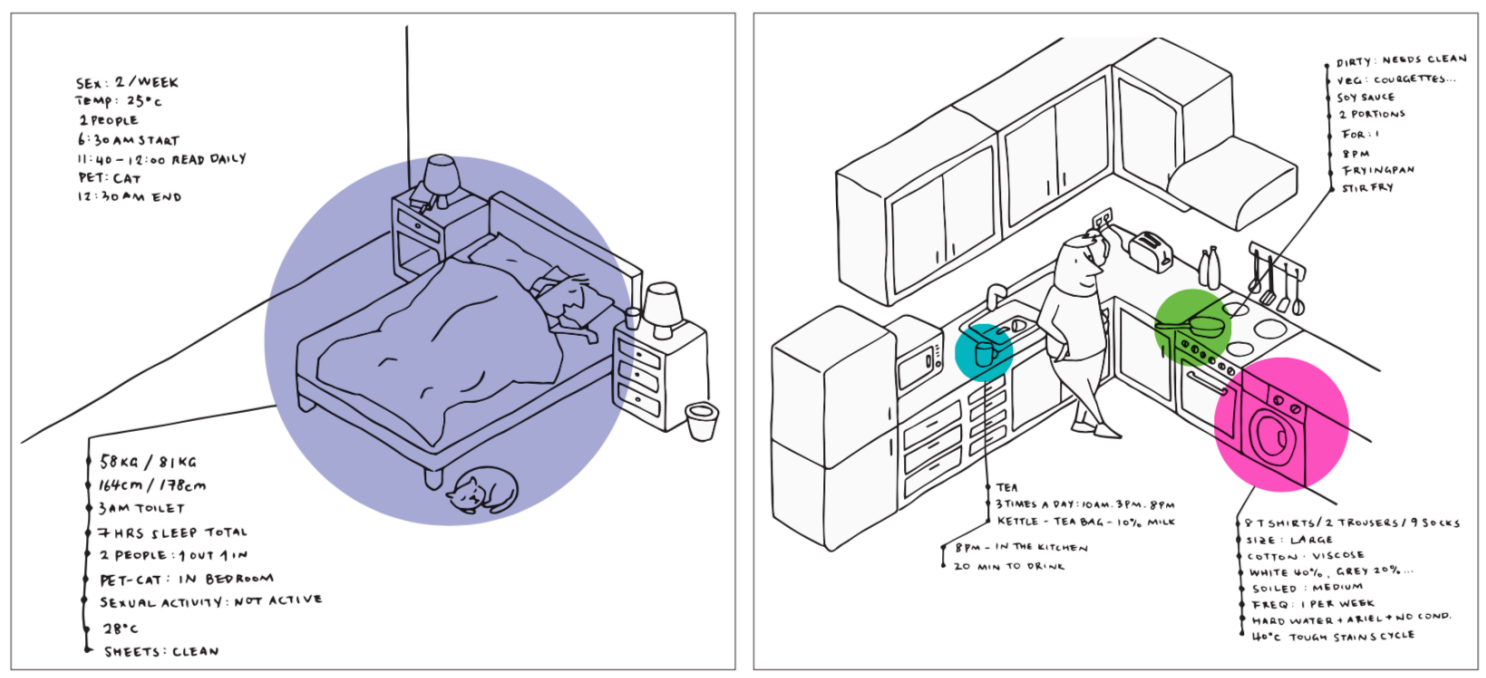

Figure 1. Two smart home scenarios presented to members of the public. 


\section{PROJECT 2: WHO'S BEHIND THE FRIDGE?}

The aim of this project was to question algorithmic logic in the context of the smart kitchen. By personally simulating an Internet-connected fridge, Delfina explored the process of being an algorithm through interaction with three participants who were already quantifying themselves with fitness- tracker wristbands. Because wearables can be seen as one of the most visible faces of IoT technology, we considered these participants to be early adopters.

To gather background data, Delfina visited participants at home for a combination dinner/interview, recording discussion about their eating habits and fitness and taking photographs of the contents of their fridges (representing potentially sensed data). Placing herself in the role of the smart algorithm, she then mined the data collected to create a fridge-report email, which she sent to each participant (without notifying them beforehand) curated around his or her personal data as well as retail trends (Figure 2). Each email incorporated possible commercial marketing strategies and employed typical user-friendly language, drawing on phrasing and style from Google and Apple's Siri. The content simulated possible outcomes of a connected fridge (e.g., suggested recipes, facts about the fridge's capabilities, advertisements, comments related to the wristband, and an Amazon shopping list). It was a mix of "big data" assumptions, real data from each participant's fridge (from photos), and qualitative data from theinterviews.

While creating the emails, taking on the role of the algorithm allowed us to see the complexities of the process. What was the best way to articulate the report: which style of language to use and what to choose in the series of decisions taken? We were forced to consider that whoever is "behind" us-in the sense of the algorithm's creators-has implications for the algorithmic outcome, and unavoidably it will reflect the incentives of commercial interests. Since the algorithms are not neutral, there are many implications for the design process. What are the social objectives of the technology? Do we care about your health and budget? How much of that do we know? Do we want to do business with you? Delfina experimented by pushing products and ordering elements in the Amazon list, for example, putting recipe ingredients automatically into the shopping list or choosing product prices (olive oil can be very expensive or very cheap).

By using participants' fridge data and by playing with what she knew from the qualitative interviews, Delfina was able to make the emails "make sense": She was accurately matching the fridge elements with the interview data. But she also created deliberate mistakes, as a way to explore how autobiographical and contextual information is otherwise missing. For example, in one email, Delfina suggested chorizo to the participant because she had sausages in her fridge, even though she knew from the context that a German friend had left those sausages, and that the participant herself very much disliked them.

If we relate this to second-order cybernetics, Delfina's experience is an example of how, in the process of attempting to personify ourselves as an algorithmic entity, as observers (in this case designers) we can never actually stand outside the situation. Delfina could not detach herself from the system that she was observing; the ideas that occurred to her as worth pursuing, with her degree of understanding, are different from those someone else might suggest. 


\section{GMail}

\section{UPDATE}

From: Your Fridge <yoursmartfridge@grnal.com>

Date: 15 December 2014 at 19:49

Subject: UPDATE

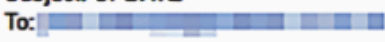

Hi John.

I am noticing the fridge is at $5.5^{\circ} \mathrm{C}$.

Milk should ideally be stored at a temperature of $4^{\circ} \mathrm{C}$ or below.

LOW on Grapefruit Juice

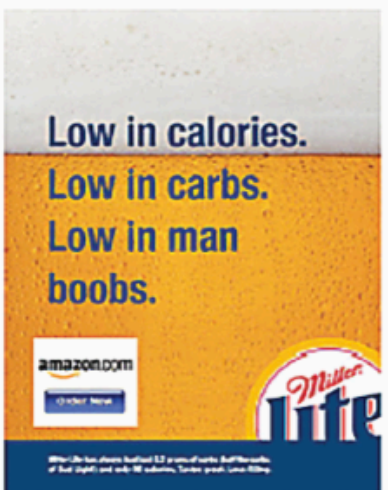

It has been a long day. May I suggest you a quick tasty meal?

A killer mac ' $n$ 'cheese

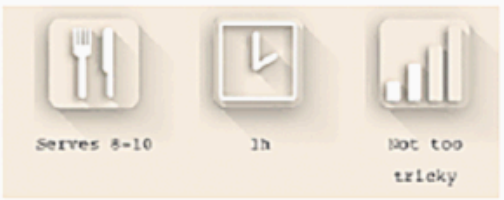

Ingredients:

sea salt

freshly ground black pepper

$45 \mathrm{~g}$ butter

3 heaped tablespoons plain flour

[CONTINUED....

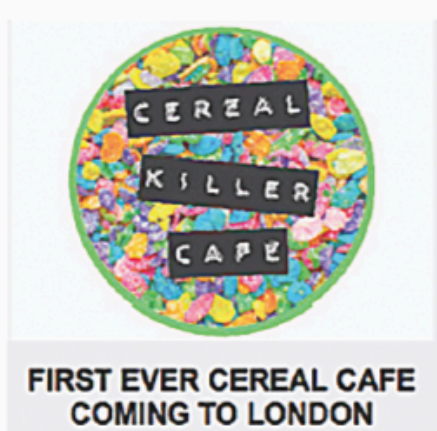

HYORATES+FUELS

YOU BETTER THAN

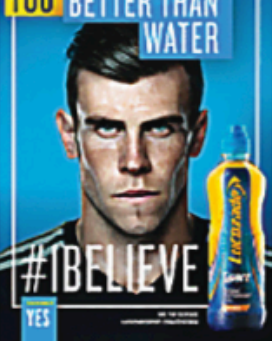

No time for shopping?

I've put together your Amazon shopping list

Tropicana Pink Grapefruit Juice $5 x £ 2.48$

Birds Eye 12 Beef Burgers $£ 4.20$

Kelloggs Frosties Cereal $£ 2.00$

Skittles Crazy Sours Pouch $4 x £ 1.00$

Rustlers Quarter Pounder Cheese $£ 2.20$

Rustlers The Deluxe With Bacon /Cheese £2.40

[CONTINOED...]

Total $£ 121.83$

Did I get it right?

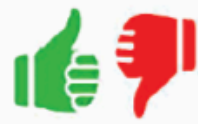

2.000 more steps to reach your goal

Good luck

Have a good evening.

Your SMART fridge

Figure 2. An example of one of the emails sent by YourSmartFridge (yoursmartfridge@gmail.com). 
Through this experiment, instead of working out the "smart" in smart home or smart fridge as a property stored in a device, we experienced a second- order cybernetics description of intelligence being a property that the observer attributes to a relationship between the system and the environment, rather than something that exists in itself.

\section{PROJECT 3: SPECULATING ONTHE IOT BACKSTAGE}

Since the IoT is characterized by being an active network of objects, humans, and the Internet, relationships are very important. Building on the "Who's behind the fridge?" experiment and its insights, we decided to explore how peopleimagine what we mightcall the IoT's backstage, in contrast to the front stage exhibited through user-friendly emails and interfaces.

Through a workshop with eight participants, representing a spectrum of technological literacy from smartphone users to those with programming experience, participants manifested the "data journeys" they imagined for an Internet-connected fridge. In a gallery space, participants were asked to imagine they had a connected fridge in their home that collects data about its contents, and to trace where they expected this data would go (or be received from). Each user started with an empty fridge and differently colored thread, which they used to connect the fridge to various entities they came across (from other participants) or to new entities they imagined (Figures 3 and 4).

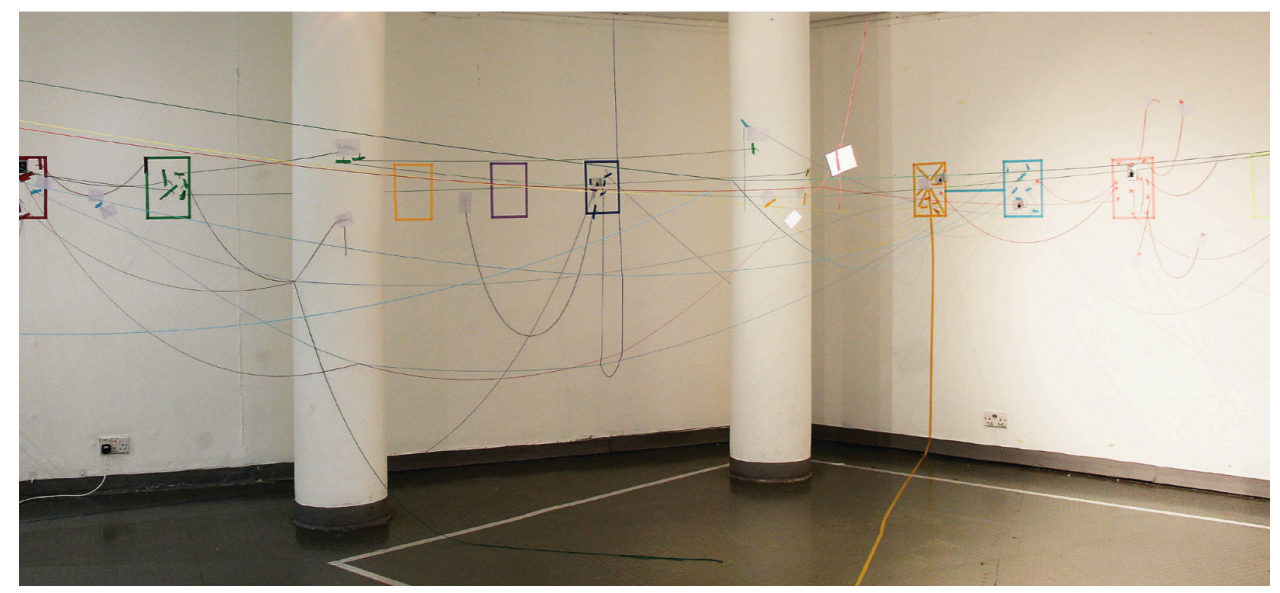

Figure 3. "Data journeys" imagined by participants, using colored threads to represent where data from the smart fridge goes. 
The outcome comprised a diversity of possible data paths and interactions, including other users, random recipes, repair services, a home hub, appliance group service, farmers, local stores, the cloud, big data, weather reports, advertising agencies, emergency services, and the doctor. The National Security Agency and Google were created as entities by different participants-and then linked together. One user even wrote at the end of a journey, "And from here who knows? Maybe up for grabs." Following the mapping, participants explained their data journeys. Interestingly, in the discussion of possible backstage scenarios they explained how their thinking had shifted as they saw and considered the journeys and entities created by others. Here again we saw the second-order principle at work: Each participant (observer) could not be detached from his or her understanding of the systems.

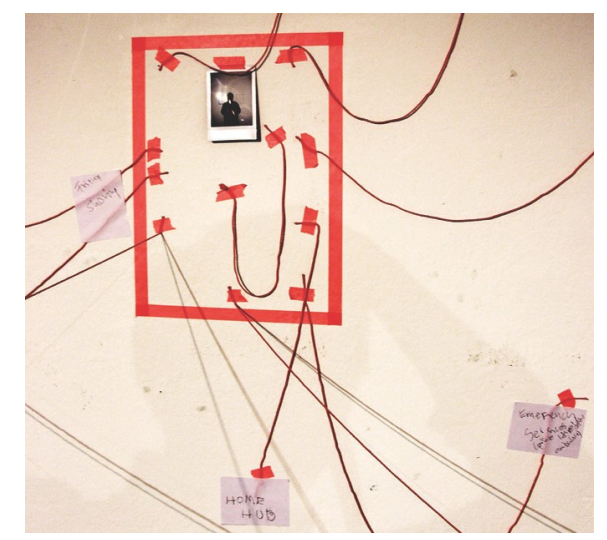

Figure 4. One participant's origins for his imagined data journeys, from his fridge to other destinations. The participant also connected to himself.

\section{FROM DATA CONSUMERS TODATA OBSERVERS}

As Paul Dourish discusses, technological practice is often regarded as a problem to be solved through searching for universal principles and decontextualized generalizations [4]. By taking second-order cybernetics as a framework and developing a series of experimental projects, we have explored a range of challenges to be considered in the design of the next generation of IoT devices and services. It became clear, particularly in the second experiment, that it was important to acknowledge relationality and context (you, the environment, and the device), subjectivity and individuality (you and your lifestyle in a nonalgorithmiclogic), and the importance of the situated meaning of our sensed data (even if habits can reveal quite a lot about your behavior, designers must consider the space and indeterminacy of the observer and his or her context).

The methods used, particularly "becoming the algorithm," offer a new way for designers working in IoT contexts to explore and challenge the assumptions behind algorithmic logic in a more experiential way, considering users as subjective constructors of meaning, and in this sense making evident the responsibility of the designers to leave the observer space for interpretation in the IoT design process. The idea of becoming the algorithm could be taken as an example to a range of contexts to research the experience of interacting with algorithmic logic from the "other" direction. 
There are further questions raised by the projects. How can the design of future technology embrace the idea of the subject as part of the algorithmic paradigm and provide a space to give interpretive agency to the user? How can it treat the construction of the self as an ongoing process (rather than an accumulation of previous data, machine learning, and big datainferences)? How can the "data journeys" be made transparent? If we define smart as a relational property, with intelligence being a property of the system rather than in the device itself, this requires a different logic from that of conventional machine learning.

How can the designer provide space to give interpretive agency to the user? What would change if we were no longer seen as consumers, but rather as active and reflective observers of our data? At present, IoT applications standardize the models of users employed: "Everything said is not said by an observer." In contrast, applying second-order cybernetics would move us from a model of detached, objective users toward seeing users as responsible participants in both data production and interpretation.

Designers have the power to frame the problems: By considering human subjectivity (therefore differences between us) and by acknowledging that we are situated, experiential, and relational subjects, there are both challenges and opportunities for designers of new generations of IoT devices and services. There is a certain uncertainty that characterizes human beings that will never be able to be covered by technology. In an IoT environment, our machines should also acknowledge their ignorance.

\section{ACKNOWLEDGMENTS}

Delfina: Special thanks to my Ph.D. tutor Ranulph Glanville (1946-2014) for his invaluable guidance during my Ph.D. journey. The title of this article is inspired by Ranulph's contribution to take into account the observer in design research and his fascination with Paul Klee's work Taking a Line for a Walk, which inspired him in his long-term interest in lines and boundaries. Also, I would like to thank Paul Pangaro for agreeing to become my external supervisor once Ranulph passed away.

1. "In a world of increasing complexity, designing digital technologies that facilitate meaningful interactions ... requires an understanding of how to design for commensurability - that is, making our ability to connect across networks commensurate with our current practices in the physical world." Giaccardi, E. Designing the connected everyday. Interactions 22, 1 (2015), 26-31.

2. Dubberly, H., Haque, U., Pangaro, P. What is interaction? Are there different types? Interactions 16, 1 (2009), 69-75; Dubberly, H., Pangaro, P. Cyberneticsand service-craft: Language for behavior- focused design. Kybernetes 36, $9 / 10$ (2007), 13011317.

3. Glanville, R. Second order cybernetics. Encyclopaedia of Life SupportSystems. EoLSS Publishers, Oxford, 2002; http:/ / www.facstaff.bucknell. edu/jvt002/brainmind/Readings/ SecondOrderCybernetics.pdf; Glanville, R. Try again. Fail again. Fail better: The cybernetics in design and the design in cybernetics. Kybernetes 36, 9/10 (2007), 1173-1206; http:/ / www.asc-cybernetics. org/systems_papers /C\%20and\%20D\%20 paper\%200670360902.pdf

4. Dourish, P. and Mainwaring, S.D. Ubicomp's colonial impulse. Proc. of Ubicomp 2012. 133-142; http: / / www. dourish.com/ publications/2012/ ubicomp2012-colonial.pdf 